\title{
Phase diagram of mixtures of hard colloidal spheres and discs: A free-volume scaled-particle approach
}

\author{
S. M. Oversteegen and H. N. W. Lekkerkerker \\ Van't Hoff Laboratory for Physical and Colloid Chemistry, Debye Institute, Utrecht University, \\ Padualaan 8, 3584CH Utrecht, The Netherlands
}

(Received 28 August 2003; accepted 6 November 2003)

\begin{abstract}
Phase diagrams of mixtures of colloidal hard spheres with hard discs are calculated by means of the free-volume theory. The free-volume fraction available to the discs is determined from scaled-particle theory. The calculations show that depletion induced phase separation should occur at low disc concentrations in systems now experimentally available. The gas-liquid equilibrium of the spheres becomes stable at comparable size ratios as with bimodal mixtures of spheres or mixtures of rods and spheres. Introducing finite thickness of the platelets gives rise to a significant lowering of the fluid branch of the binodal. (C) 2004 American Institute of Physics.
\end{abstract}

[DOI: $10.1063 / 1.1637573$ ]

\section{INTRODUCTION}

Mixtures of colloidal particles that differ in size and shape are ubiquitous in industry, food science, and the biological realm. ${ }^{1,2}$ Knowledge of the phase diagram of those systems is essential to understand their stability. A range of colloidal mixtures have been studied extensively by means of experiments, simulations, and theory. Especially mixtures of hard spheres with polymers (see Ref. 3, and references therein) or hard smaller spheres obtained much attention (see Ref. 4, and references therein). In such systems phase transitions may take place for purely entropic reasons, i.e., the species may gain conformational entropy when they are no longer hindered by the other species which are consequently expelled. This so-called depletion effect ${ }^{5,6}$ is even more pronounced in anisometric mixtures of, e.g., rods and spheres. ${ }^{7-9}$ Here we determine the phase behavior of anisometric mixtures of platelets and spheres by means of the free-volume theory. ${ }^{10}$ This theory accounts for the volume in the system that is available to the species in a hypothetical reservoir and has previously proven to be successful to bimodal mixtures of spheres ${ }^{11}$ and to mixtures of rods and spheres. ${ }^{12,13}$

\section{FREE-VOLUME THEORY}

Consider a system of $N_{s}$ hard spheres with diameter $\sigma$ in a volume $V$ at temperature $T$. Suppose this system is in thermodynamic equilibrium with a reservoir of platelets with a diameter $D$ and thickness $L$. The appropriate thermodynamic quantity to describe this system is the semigrand potential. Applying Widom's insertion theorem ${ }^{14}$ and elementary thermodynamic relations, one obtains for dilute platelet suspensions ${ }^{10-13}$

$$
\Omega\left(N_{s}, \mu_{p}, V, T\right)=A^{\circ}\left(N_{s}, V, T\right)-p^{R} \alpha V .
$$

Here $A^{\circ}$ is the Helmholtz energy of the unperturbed hard sphere system and $\alpha$ is the so-called free-volume fraction, i.e., the relative amount of the volume $V$ that is accessible to the platelets. A convenient expression for the pressure $p^{R}$ exerted by the reservoir of platelets is given by ${ }^{15}$

$$
\frac{p^{R}}{\rho_{p}^{R} k_{B} T}=\frac{1}{1-\phi_{p}^{R}}+\frac{3 \kappa \phi_{p}^{R}}{\left(1-\phi_{p}^{R}\right)^{2}}+\frac{\kappa^{2} \phi_{p}^{R^{2}}\left(3-\phi_{p}^{R}\right)}{\left(1-\phi_{p}^{R}\right)^{3}} .
$$

Here $\phi_{p}^{R}$ is the volume fraction of discs in the reservoir. The nonsphericity parameter $\kappa$ follows from the second virial coefficient $B_{2}$ and the platelet's volume $v_{p}$ according to

$$
\kappa=\frac{B_{2} / v_{p}-1}{3} .
$$

Upon comparison with simulations ${ }^{17,18}$ Eq. (2) is very accurate for both platelets of finite thickness as well as discs. The mathematics in the following is substantially simplified if we take discs, i.e., infinitely thin platelets, without losing the physics. The second virial coefficient then simply reads

$$
B_{2}=\pi^{2} D^{3} / 16
$$

which converts Eq. (2) to ${ }^{19}$

$\frac{p^{R} v_{s}}{k_{B} T}=\frac{\pi}{6 q^{3}}\left[\left(\rho_{p}^{R} D^{3}\right)+\left(\frac{\pi^{2}}{16}\right)\left(\rho_{p}^{R} D^{3}\right)^{2}+\left(\frac{\pi^{4}}{768}\right)\left(\rho_{p}^{R} D^{3}\right)^{3}\right]$,

where we introduce the aspect ratio $q \equiv D / \sigma$ and the discs' number density in the reservoir, $\rho_{p}^{R}$.

Coexistence between phases $\alpha$ and $\beta$ at a given reservoir pressure is found from

$$
p^{\alpha}\left(\phi_{s}^{\alpha}\right)=p^{\beta}\left(\phi_{s}^{\beta}\right), \quad \mu_{s}^{\alpha}\left(\phi_{s}^{\alpha}\right)=\mu_{s}^{\beta}\left(\phi_{s}^{\beta}\right) .
$$

We obtain the pressure of the system and chemical potential of the spheres from Eq. (1) as

$$
p=-\left(\frac{\partial \Omega}{\partial V}\right)_{T, N_{s}, \mu_{p}}=p^{\circ}+p^{R}\left[\alpha-\phi_{s}\left(\frac{\partial \alpha}{\partial \phi_{s}}\right)\right]
$$




$$
\mu_{s}=\left(\frac{\partial \Omega}{\partial N_{s}}\right)_{V, T, \mu_{p}}=\mu_{s}^{\circ}-p^{R} v_{s}\left(\frac{\partial \alpha}{\partial \phi_{s}}\right) .
$$

Here $\phi_{s}=N_{s}(\pi / 6) \sigma^{3} / V$ is the volume fraction of the spheres. The pressure and chemical potential of the unperturbed hard-sphere fluid are found from the CarnahanStarling equation ${ }^{20}$

$$
\begin{aligned}
\frac{p^{\circ} v_{s}}{k_{B} T} & =\frac{\phi_{s}\left(1+\phi_{s}+\phi_{s}^{2}-\phi_{s}^{3}\right)}{\left(1-\phi_{s}\right)^{3}}, \\
\frac{\mu_{s}^{\circ}}{k_{B} T} & =\ln \phi_{s}+\frac{\phi_{s}\left(8-9 \phi_{s}+3 \phi_{s}^{2}\right)}{\left(1-\phi_{s}\right)^{3}} .
\end{aligned}
$$

The equation of state of the hard-sphere crystal is given by $\mathrm{Wood}^{21}$

$$
\frac{p^{\circ} v_{s}}{k_{B} T}=\frac{3 \phi_{s} \phi_{\mathrm{cp}}}{\phi_{\mathrm{cp}}-\phi_{s}}
$$

where $\phi_{\mathrm{cp}}=\pi \sqrt{2} / 6 \approx 0,74$ is the closed packed density. The chemical potential follows from integration of the Maxwell relation $\left(\partial p / \partial N_{s}\right)_{T}=-\left(\partial \mu_{s} / \partial V\right)_{T}$,

$$
\frac{\mu_{s}^{\circ}}{k_{B} T}=2.1306+3 \ln \left(\frac{\phi_{s} \phi_{\mathrm{cp}}}{\phi_{\mathrm{cp}}-\phi_{s}}\right)+\frac{3 \phi_{\mathrm{cp}}}{\phi_{\mathrm{cp}}-\phi_{s}} .
$$

The first term on the right-hand side is an integration constant derived from the absolute free energy of a hard sphere crystal from Monte Carlo simulations at $\phi_{s}=0.576 .{ }^{22}$

\section{SCALED-PARTICLE THEORY}

The free-volume fraction $\alpha$ is determined from the chemical potential of the discs. Inserting a disc in a sea of spheres adds, next to the mixing entropy, a work term $W$ to that chemical potential

$$
\mu_{p}=\mu_{p}^{\circ}+k_{B} T \ln \rho_{p}+W .
$$

On the other hand, it follows from Widom's insertion theorem ${ }^{14}$ that

$$
\mu_{p}=\mu_{p}^{\circ}+k_{B} T \ln \rho_{p}-k_{B} T \ln \alpha .
$$

The required work to insert a disc in between spheres therefore amounts to $W=-k_{B} T \ln \alpha$ or, alternatively,

$$
\alpha=e^{-W / k_{B} T} .
$$

Looking for the free volume fraction $\alpha$ is therefore equivalent to finding an expression for $W$. In order to determine the insertion work $W$, the scaled-particle theory considers two size limits. To that end, we scale the disc diameter $D$ with a parameter $\lambda$. Since the disc is infinitely thin, the thickness does not need to be scaled.

For the limit $\lambda \ll 1$ the small discs may be considered to be points and the overlap volume between the spheres is therefore negligible. Hence, in that limit the volume available to the disc is $V-N_{s} \omega(\lambda D, \sigma)$, where $\omega(\lambda D, \sigma)$ is the volume of a sphere of diameter $\sigma$ that is excluded to a disc. Thus, the free-volume fraction is $\alpha=1-\rho_{s} \omega(\lambda D, \sigma)$. Using Eq. (15) it follows that

$$
W(\lambda \ll 1)=-k_{B} T \ln \left(1-\rho_{s} \omega(\lambda D, \sigma)\right) .
$$

On the other hand, if $\lambda \gg 1$, the work required to insert a large disc between the spheres, will approximately be the volume work to create a hole with the size of a disc; $W \approx p v_{p}$. However, since the discs are infinitely thin, it applies that the size of a disc vanishes; $v_{p}=0$. Hence,

$$
W(\lambda \gg 1)=p v_{p}=0 .
$$

This rather remarkable result also applies to infinitely thin rods. ${ }^{12,23}$

The essence of the scaled-particle theory is that we may add both limits, where we expand $W$ in the limit $\lambda \ll 1$ around $\lambda=0$. From Eqs. (16) and (17) we obtain

$$
\begin{aligned}
W(\lambda)= & W(0)+\left(\frac{\partial W}{\partial \lambda}\right)_{\lambda=0} \lambda+\frac{1}{2}\left(\frac{\partial^{2} W}{\partial \lambda^{2}}\right)_{\lambda=0} \lambda^{2}+p v_{p} \\
= & -k_{B} T \ln \left(1-\rho_{s} \omega_{0}\right)+\frac{k_{B} T \rho_{s}}{1-\rho_{s} \omega_{0}} \omega_{0}^{\prime} \lambda \\
& +\frac{1}{2}\left(\frac{k_{B} T \rho_{s}}{1-\rho_{s} \omega_{0}} \omega_{0}^{\prime \prime}+\frac{k_{B} T \rho_{s}^{2}}{\left(1-\rho_{s} \omega_{0}\right)^{2}} \omega_{0}^{\prime 2}\right) \lambda^{2},
\end{aligned}
$$

where $\omega_{0}, \omega_{0}^{\prime}$, and $\omega_{0}^{\prime \prime}$ are the excluded volume, its first and second derivatives with respect to $\lambda$, respectively, evaluated for $\lambda=0$.

When we finally scale the disc to the desired size by putting $\lambda=1$, we find from Eq. (18) using Eq. (15) for the free-volume fraction

$$
\begin{aligned}
\alpha= & \left(1-\rho_{s} \omega_{0}\right) \exp \left[-\left(\omega_{0}^{\prime}+\frac{1}{2} \omega_{0}^{\prime \prime}\right)\left(\frac{\rho_{s}}{1-\rho_{s} \omega_{0}}\right)\right. \\
& \left.-\frac{1}{2} \omega_{0}^{\prime 2}\left(\frac{\rho_{s}}{1-\rho_{s} \omega_{0}}\right)^{2}\right] .
\end{aligned}
$$

The problem has now reduced to finding the excluded volume of a disc around a sphere. Let us place a disc into a certain orientation and move it in such a way around a sphere, that its center is as close as possible to the sphere. In this way we probe the volume that is inaccessible to the disc. A different orientation of the disc relative to the sphere is equivalent as redefining the coordinate system. In this way it is easily seen that all orientations yield the same excluded volume. For a disc of diameter $D$ around a sphere of diameter $\sigma$ we find from the body of revolution of a line probing a circle

$$
\begin{aligned}
\omega & =2 \pi \int_{0}^{\sigma / 2}\left(\frac{1}{2} D+\sqrt{\frac{1}{4} \sigma^{2}-y^{2}}\right)^{2} \mathrm{~d} y \\
& =\frac{\pi}{4} D^{2} \sigma+\frac{\pi^{2}}{8} D \sigma^{2}+\frac{\pi}{6} \sigma^{3} .
\end{aligned}
$$

This is the same result as found for spheres around a disc. ${ }^{19}$ Scaling the diameter of the disc with $\lambda$, we find for the coefficients in Eq. (19),

$$
\begin{aligned}
& \omega_{0}=\frac{\pi}{6} \sigma^{3}=v_{s}, \quad \omega_{0}^{\prime}=\frac{\pi^{2}}{8} D \sigma^{2}=\frac{3}{4} \pi q v_{s}, \\
& \omega_{0}^{\prime \prime}=\frac{\pi}{2} D^{2} \sigma=3 q^{2} v_{s} .
\end{aligned}
$$



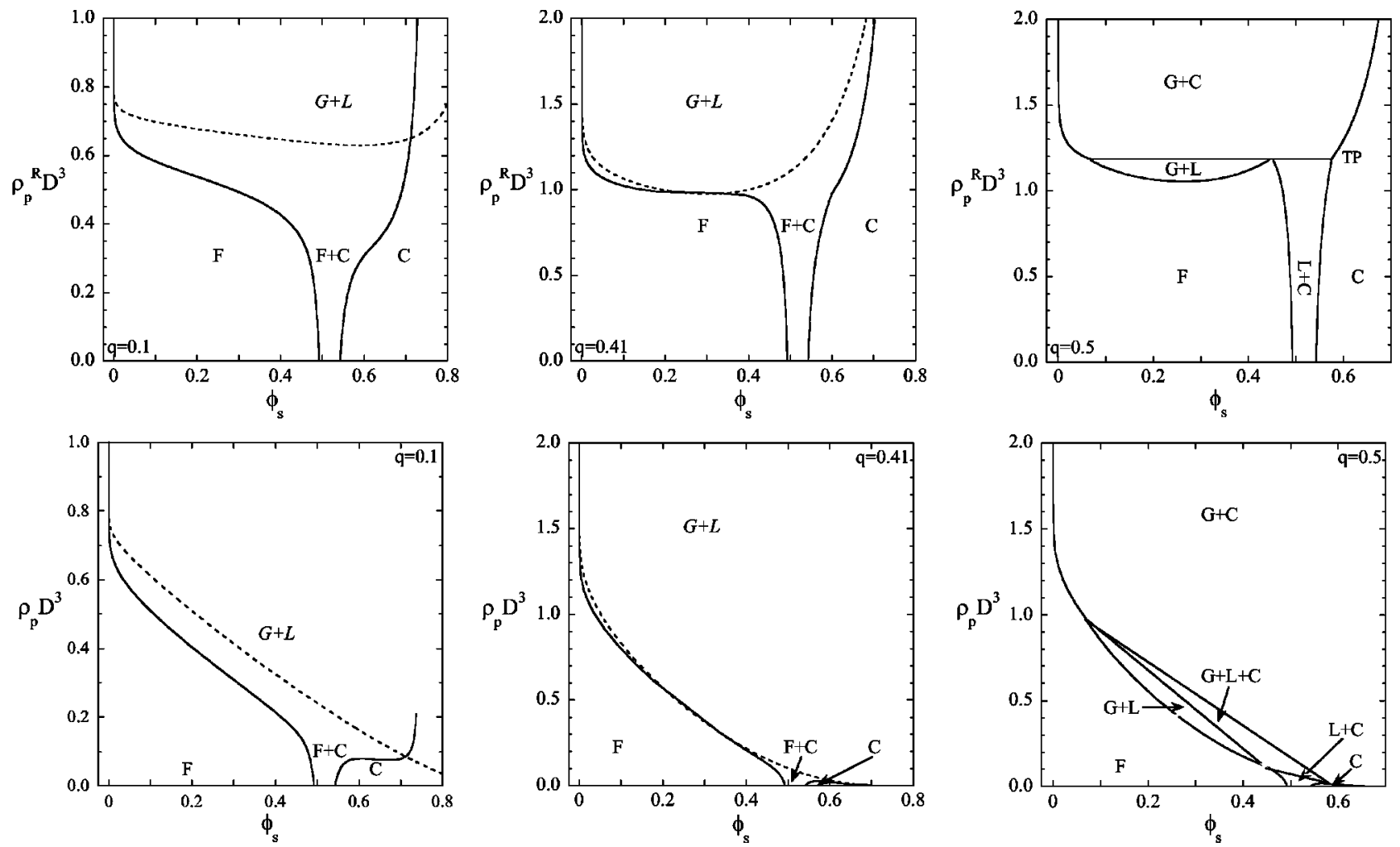

FIG. 1. Calculated phase diagram for mixtures of hard spheres (volume fraction $\phi_{s}$ ) with hard discs. The upper row as a function of the reduced reservoir number density $\rho_{p} D^{3}$, where $D$ is the disc's diameter, in the second row the corresponding reduced number density in the system. Dashed lines show the metastable gas-liquid equilibrium.

Here we introduced the aspect ratio $q=D / \sigma$. Using $\phi_{s}$ $=\rho_{s} v_{s}$, the free-volume fraction, Eq. (19), finally reads

$$
\begin{aligned}
\alpha= & \left(1-\phi_{s}\right) \exp \left[-\left\{A\left(\frac{\phi_{s}}{1-\phi_{s}}\right)+B\left(\frac{\phi_{s}}{1-\phi_{s}}\right)^{2}\right.\right. \\
& \left.\left.+C\left(\frac{\phi_{s}}{1-\phi_{s}}\right)^{3}\right\}\right],
\end{aligned}
$$

with

$$
A=\frac{3}{2} q\left(\frac{\pi}{2}+q\right), \quad B=\frac{9 \pi^{2}}{32} q^{2}, \quad C=0 .
$$

Since we consider dilute disc dispersions, the chemical potential of the reservoir may be given as $\mu_{p}^{R}=\mu_{p}^{\circ}$ $+k_{B} T \ln \rho_{p}^{R}$. In equilibrium applies $\mu_{p}=\mu_{p}^{R}$. Using Eq. (14), we find the volume fraction of discs in the system as $\rho_{p}=\alpha \rho_{p}^{R}$.

\section{RESULTS}

At a given number density, $\rho_{p}^{R}$ of discs in the reservoir, the pressure of the reservoir on the system follows from Eq. (5). Together with Eqs. (22) and (23) then follows the perturbation on the pure hard spheres due to the presence of the discs in Eqs. (7) and (8).

The set equations of Eq. (6) can be solved numerically using Eqs. (9) and (10) in Eqs. (7) and (8), respectively, for a given $\rho_{p}^{R}$. This set gives the gas-liquid coexistence of a "liquid phase" rich in spheres (poor in discs) and a "gas phase" that is poor in spheres (relatively rich in discs). We calculated points down to the critical point which can be found numerically from the roots of the first and second derivatives of the pressure, Eq. (7). If we use Eqs. (9) and (10) for phase $\alpha$ and Eqs. (11) and (12) for phase $\beta$, the fluid-crystal equilibrium can be found from the set equations of Eq. (6) at given $\rho_{p}^{R}$. Results are given in Fig. 1.

Since the phase transition takes place at $\rho_{p}^{R} D^{3} \sim 1$, no isotropic-nematic transition of discs is expected. Simulations ${ }^{16,17}$ show that this is first found at a density $\rho_{p}^{R} D^{3} \sim 4$. For relatively small discs (low $q$ ) the gas-liquid equilibrium $(G+L)$ is located inside the $F+C$ region and is metastable, indicated by the dashed lines in Fig. 1. For large enough discs $(q \equiv D / \sigma \gtrsim 0.41) G+L$ becomes stable, which leads to the existence of a triple line. This aspect ratio is comparable to those found for polymer-spheres mixtures $[q=0.4$ (Ref. 24)] and (infinitely thin) $\operatorname{rods}[q=0.3$ (Refs. $12,13)]$.

Experimentally the number density of discs in the system rather that in the (hypothetical) reservoir is relevant. The calculated phase diagrams in the upper row of Fig. 1 are therefore transformed to experimentally accessible diagrams in the second row by $\rho_{p}=\alpha \rho_{p}^{R} \cdot{ }^{10}$ This forces the horizontal tie lines to be slanted. As a consequence the triple line opens up as a triangle.

In an experiment the platelets have a finite thickness. It seems reasonable as a first approximation to apply the theory for infinitely thin platelets to the experimental system given in Fig. 2(a). The silica spheres have a diameter of $\sigma$ $=700 \mathrm{~nm}$, whereas the silica coated gibbsite platelets are $L$ 


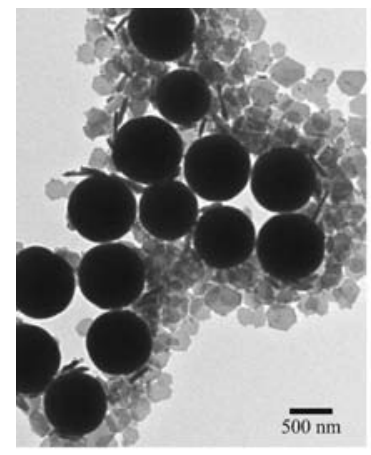

(a)

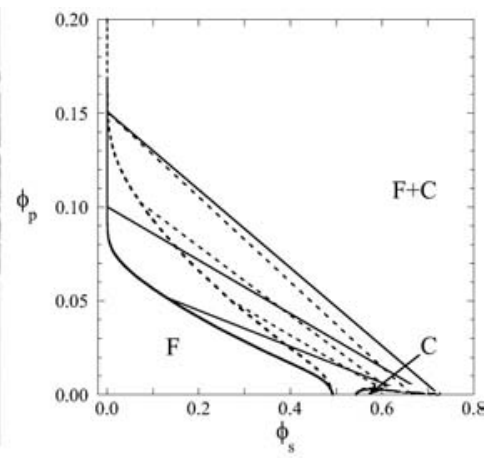

(b)
FIG. 2. (a) TEM micrograph of a mixture of silica spheres $(\sigma=700 \mathrm{~nm})$ with silica coated gibbsite plates $(D=200 \mathrm{~nm}, L=30 \mathrm{~nm})$. (b) The calculated phase diagram of the experimental system. The dashed lines give the phase diagram from discs where the volume fraction of platelets, $\phi_{p}$, is determined from the reduced number density for discs via Eq. (24). Upon replacing both the second virial coefficient in Eq. (2) as well as the coefficients of the free volume fraction in Eq. (22) by those for thin cylinders, the solid lines are found.

$=30 \mathrm{~nm}$ thick and $D=200 \mathrm{~nm}$ wide. The reduced number density can be converted to a volume fraction via

$$
\phi_{p}=\rho_{p} v_{p}=\rho_{p} \frac{\pi}{4} D^{2} L=\rho_{p} D^{3} \frac{\pi}{4} \frac{L}{D} .
$$

For the experimental size ratio $(q=2 / 7<0.41)$ the liquidgas phase transition is metastable, so only the fluid-crystal phase boundary and tie lines are given by the dashed lines in Fig. 2(b).

It is straightforward to extend the above theory to platelets of diameter $D$ with a finite thickness $L$ represented by short cylinders. The second virial coefficient of a reservoir of cylinders reads ${ }^{25}$

$$
B_{2}=\frac{\pi^{2} D^{3}}{16}+\frac{\pi D^{2} L}{8}(3+\pi)+\frac{\pi D L^{2}}{4} .
$$

For infinitely thin platelets $(L=0)$ Eq. (25) reduces to Eq. (4). It can be shown that Eq. (2), using the second virial coefficient in Eq. (3) from Eq. (25), gives the same pressure as from simulations ${ }^{18}$ up to densities $\rho_{p}^{R} D^{3} \lesssim 2.0$. That is, Eq. (2) using Eq. (25) suffices for our present purposes.

By applying the scaled particle procedure of Sec. III to a sphere probing the volume around a cylinder, we find for the free volume fraction the same functional form as Eq. (22) now with the coefficients

$$
\begin{aligned}
& A=\frac{3}{2} q\left[\frac{\pi}{2}+q+r(1+q)^{2}\right], \\
& B=\frac{9}{8} q^{2}\left[\left(\frac{\pi}{2}+r\right)^{2}+4 q r\right], \\
& C=\frac{9}{2} q^{3} r .
\end{aligned}
$$

Here we defined $r \equiv L / D$. It is easily seen that for discs $(r=0)$ Eq. (26) reduces to Eq. (23).
Inserting Eqs. (25) and (26) into Eqs. (2) and (22), respectively, and going through the above procedure using the experimental system of Fig. 2(a), we find the phase diagram given by the solid line in Fig. 2(b). Clearly, the fluid branch of the binodal goes down by almost a factor 2, whereas from the tie lines we see that the crystal phase tend to shift to higher volume fractions of the spheres. Hence, introducing finite thickness to the platelets leads to a significant lowering of the binodal. This can also be observed for rods when infinitely thin needles are replaced by spherocylinders. ${ }^{12,13}$

If we prepare samples of $\phi_{s}=0.025$ spheres, we may already expect crystalline ordering of the spheres at $\phi_{p}$ $=0.07$. This volume fraction is one order of magnitude larger than experimentally found for dispersions of rods in spheres $\left(\phi_{\text {rod }}=0.005\right.$ for a similar aspect ratio $\left.{ }^{9}\right)$ but one order of magnitude smaller than predicted for binary hard spheres mixtures (we estimate $\phi_{\text {small sphere }}>0.3$ for a comparable size ratio ${ }^{26,27}$ ). We predict that fairly low platelet concentrations suffice to induce phase separation. We hope to address this experimentally for systems as shown in Fig. 2.

\section{ACKNOWLEDGMENTS}

We are grateful to Dr. J. E. G. J. Wijnhoven for providing us with the synthesis and TEM-picture of the mixture. H. H. Wensink is thanked for fruitful discussions. This work is part of the SoftLink research program of the "Stichting voor Fundamenteel Onderzoek der Materie (FOM)," which is financially supported by the "Nederlandse Organisatie voor Wetenschappelijk Onderzoek (NWO)."

${ }^{1}$ W.B. Russel, D.A. Saville, and W.R. Schowalter, Colloidal Dispersions (Cambridge University Press, Crambridge, 1989).

${ }^{2}$ D.F. Evans and H. Wennerström H., The Colloidal Domain: Where Physics, Chemistry, Biology, and Technology Meet (Wiley-VCH, New York, 1999).

${ }^{3}$ R. Tuinier, J. Rieger, and C.G. de Kruif, Adv. Colloid Interface Sci. 103, 1 (2003).

${ }^{4}$ M. Dijkstra, R. van Roij, and R. Evans, Phys. Rev. E 59, 5744 (1999).

${ }^{5}$ S. Asakura and F. Oosawa, J. Chem. Phys. 22, 1255 (1954).

${ }^{6}$ A. Vrij, Pure Appl. Chem. 48, 471 (1976).

${ }^{7}$ M. Adams, Z. Dogic, S.L. Keller, and S. Fraden, Nature (London) 393, 349 (1998).

${ }^{8}$ G.H. Koenderink, G.A. Vliegenthart, S.G.J.M. Kluijtmans, A. van Blaaderen, A.P. Philipse, and H.N.W. Lekkerkerker, Langmuir 15, 4693 (1999).

${ }^{9}$ G.A. Vliegenthart, A. van Blaaderen, and H.N.W. Lekkerkerker, Faraday Discuss. 112, 173 (1999).

${ }^{10}$ H.N.W. Lekkerkerker, W.C.K. Poon, P.N. Pusey, A. Stroobants, and P.B. Warren, Europhys. Lett. 20, 559 (1992).

${ }^{11}$ H.N.W. Lekkerkerker and A. Stroobants, Physica A 195, 387 (1993).

${ }^{12}$ P.G. Bolhuis and D. Frenkel, J. Chem. Phys. 101, 9869 (1994).

${ }^{13}$ G.A. Vliegenthart and H.N.W. Lekkerkerker, J. Chem. Phys. 111, 4153 (1999).

${ }^{14}$ B. Widom, J. Chem. Phys. 39, 2808 (1963).

${ }^{15}$ T. Boublik and D. Nezbeda, Collect. Czech. Chem. Commun. 51, 2301 (1985).

${ }^{16}$ D. Frenkel and R. Eppenga, Phys. Rev. Lett. 49, 1089 (1982).

${ }^{17}$ R. Eppenga and D. Frenkel, Mol. Phys. 52, 1303 (1984).

${ }^{18}$ S.D. Zhang, P.A. Reynolds, and J.S. van Duijneveldt, J. Chem. Phys. 117, 9947 (2002)

${ }^{19}$ S.D. Zhang, P.A. Reynolds, and J.S. van Duijneveldt, Mol. Phys. 100, 3041 (2002).

${ }^{20}$ N.F. Carnahan and K.E. Starling, J. Chem. Phys. 51, 635 (1969).

${ }^{21}$ W.W. Wood, J. Chem. Phys. 20, 1334 (1952).

${ }^{22}$ D. Frenkel and J.C. Ladd, J. Chem. Phys. 81, 3188 (1984). 
${ }^{23}$ H. Reiss, H.L. Frisch, and J.L. Lebowitz, J. Chem. Phys. 31, 369 (1959). ${ }^{24}$ M. Dijkstra, J.M. Brader, and R. Evans, J. Phys.: Condens. Matter 11, 10079 (1999).

${ }^{25}$ L. Onsager, Ann. N.Y. Acad. Sci. 51, 627 (1949).
${ }^{26}$ J.S. van Duijneveldt, A.W. Heinen, and H.N.W. Lekkerkerker, Europhys. Lett. 21, 369 (1993).

${ }^{27}$ P.D. Kaplan, J.L. Rouke, A.G. Yodh, and D.J. Pine, Phys. Rev. Lett. 72, 582 (1994). 